\title{
Multi-objective optimization and data analysis in informationization
}

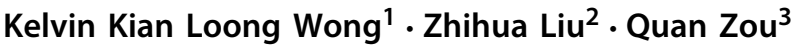

Published online: 19 April 2019

(c) Springer-Verlag GmbH Austria, part of Springer Nature 2019

In the information technology age, informationization is the inevitable development trend of the industries pertaining to medical, social, manufacturing, or engineering fields. From the individual information query to the allocation of thousands of resources and staff, all the information operations involved is a part of the process of informationization. In the past few decades, the computing hardware and software has continued to grow exponentially. Furthermore, breakthrough in operational research and arrival of the era of big data has helped us to find a better solution for human information needs. However, there still are some challenging problems for providing a better service. For most given problems, multi-objective optimization and data mining can provide the optimal solution. And machine learning such as artificial neural network, deep learning, evolutionary algorithm, and genetic algorithm are some of the other well-established techniques we can explore for solutions generation. In the typical industry, using these algorithms can help organizations find the potential factors which affect their customers more accurately and improve service provision. It can also identify trends that bridge the gaps among fragments of seemingly unrelated information. In addition, the process of informationtization also promotes the development of informatics, including membrane computing, gene expressions, genetic computing, etc. These new technologies can offer much higher quality and personalized service for people.

Multi-objective optimization and data analysis techniques that are related to informationization have been applied in major fields of engineering and management pertaining to resource allocation and planning. In particular, informationization has appeared in industries such as agriculture, education, etc. Furthermore, computer vision and imaging is used for data analysis and health diagnostics in the medical industry, and also for structural analysis in the manufacturing industry. In this edito-

Kelvin Kian Loong Wong

kelvin.wong@adelaide.edu.au

1 School of Electrical and Electronic Engineering, University of Adelaide, Adelaide, SA, Australia

2 Department of Biostatistics, Harvard School of Public Health, Boston, USA

3 Institute of Fundamental and Frontier Sciences, University of Electronic Science and Technology of China, Chengdu, China 
rial preface, we also present the application of informationization in the health and pharmaceutical industry. In particular, bioinformatics that relies heavily on information analysis is used in the discovery of new drugs and cure for diseases. As presented, this special issue collected a series of articles that highlight the importance of informationization in the various areas of research. This issue has 13 research articles on the applications of resource management and planning, education, computer vision, biomedical imaging, manufacturing, and bioinformatics. Details of these papers are as follows.

Industrial management based on informationization has been used widely in resource allocation and planning. This group of three papers investigate resource management and planning. The paper provided by Goli et al. was entitled "A multi-objective invasive weed optimization algorithm for robust aggregate production planning under uncertain seasonal demand". In this study, the authors introduced a robust multi-objective multi-period aggregate production planning (APP) problem based on different scenarios under uncertain seasonal demand.

The paper provided by Ruan et al. was entitled "Effective learning model of user classification based on ensemble learning algorithms". They proposed a hybrid learning model based ensemble learning algorithms for recognizing user to be sensitive to electric charge. On the basis of experiment employing real data, it is proved that learning model has high performance.

The paper entitled "Multi-objective multi-mode resource constrained project scheduling problem using Pareto-based algorithms" was provided by Tirkolaee et al. In this study, the authors proposed a non-linear programming model to formulate the resource-constrained project scheduling problem. The obtained results demonstrate that multi-objective simulated annealing algorithm has better efficiency in small-sized problems and non-dominated sorting genetic algorithm II in large-sized problems.

Next, we also have a group of articles pertaining to education resource management. The paper provided by Li et al. was entitled "Improved randomized learning algorithms for imbalanced and noisy educational data classification". In this study, the authors investigated a novel type of cost function that focuses on the combined effects of class imbalance and class noise to improve randomized learning algorithms. Their experimental study on several educational data classification tasks have verified the advantages of the proposed algorithms.

The paper entitled "Learning performance prediction via convolutional GRU and explainable neural networks in e-learning environments" entitled was provided by Wang et al. They developed a predictive model for effective learning feature extracting, learning performance predicting and result reasoning, in order to ensure that learners' potential problems can be identified as early as possible. Their results show that the proposed approach performs favorably compared with several other state-of-the-art methods.

We also explore the frameworks for computer vision applied in industry. The paper provided by Yang et al. was entitled "Adaptive Weber-face for robust illumination face recognition". In this study, the authors presented an adaptive Weber-face method to deal with varying lighting and to extract illumination-insensitive representation for face recognition. The experiments demonstrate that the adaptive Weber-face method achieves the high recognition rates. 
The paper provided by Lai et al. was entitled by "A novel non-parametric transform stereo matching method based on mutual relationship". In this study, a novel non-parametric transform stereo matching method based on mutual relationship is proposed, in order to cope with the problem of the vast majority local stereo matching approaches.

Computer vision and imaging can be implemented in medical image diagnosis, which we present in the following set of papers. The paper provided by Zhang et al. was entitled by "Deep learning based vein segmentation from susceptibilityweighted images". The authors proposed an end-to-end joint learning framework for artery-specific coronary calcification identification in non-contrast cardiac CT. The quantitative results indicate that the new method can be used as a reliable clinical diagnostic tool for coronary calcification identification.

The paper entitled "A novel machine-learning algorithm to estimate the position and size of myocardial infarction for MRI sequence" was provided by Zhang et al. In this study, they presented a susceptibility-weighted imaging (SWI) vein segmentation network which consists of 19 convolutional blocks to extract discriminative features for dense prediction. The proposed method provided a potential tool to quantify veins in SWIs with accuracy to assist decision making especially for thrombolytic therapy.

The paper provided by $\mathrm{Xu}$ et al. was entitled "An end-to-end joint learning framework of artery-specific coronary calcium scoring in non-contrast cardiac CT". They proposed a machine-learning and image motion statistic based approach to automating the detection and localization of myocardial infarction area. The field experiments showed that their method can lead to a promising diagnostic support tool to assists clinicians, particularly for novice readers with limited experience.

Furthermore, we have an article that implemented imaging for structural health monitoring, which pertains to the manufacturing industry. This paper was entitled "Adaptive sparse reconstruction of damage localization via Lamb waves for structure health monitoring". In this study, Zhang et al. applied sparse reconstruction method for damage localization via ultrasonic Lamb waves in structure health monitoring, in order to solve the problems of noise and unknown sparsity on signal reconstruction.

Bioinformatics is an increasing important field and can also be used to investigate health issues. The paper provided by Wang et al. was entitled by "Identification of hormone-binding proteins using a novel ensemble classifier". Correct identification of Hormone-binding proteins is important for their future research direction and for the coordination of hormones. Their results indicated that $90.6 \%$ of accuracy was achieved in five-fold cross validation, which demonstrate that the methods was superior to that of other published methods.

The paper entitled by "Multidimensional scaling method for prediction of lysine glycation sites" was provided by Li et al. In this study, the authors proposed a novel predictor named MDS_GlySitePred to predict lysine glycation sites and support vector machine algorithm. Their experimental results indicated that MDS_GlySitePred significantly outperforms four existing glycation site predictors.

As presented, this issue collected a series of articles that highlight the importance of informationization in the various fields of computing research. We hope this special issue will provide a platform for researchers to collaborate on some research areas with the aim of promoting scientific studies in relation to multi-objective optimization and 
data analysis. In addition, it can be used as a valuable source of references for computer science researchers to conduct more advanced studies in information technology.

Publisher's Note Springer Nature remains neutral with regard to jurisdictional claims in published maps and institutional affiliations. 